\title{
Long non-coding RNA CASC15 is upregulated in hepatocellular carcinoma and facilitates hepatocarcinogenesis
}

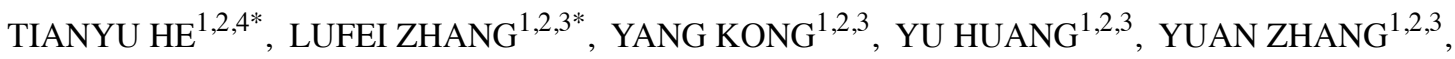

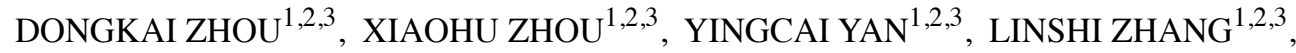 \\ SINAN LU ${ }^{1,2,3}$, JIARONG ZHOU ${ }^{1,2,3}$ and WEILIN WANG ${ }^{1,2,3}$
}

\begin{abstract}
${ }^{1}$ Key Laboratory of Precision Diagnosis and Treatment for Hepatobiliary and Pancreatic Tumor of Zhejiang Province, First Affiliated Hospital, School of Medicine, Zhejiang University, Hangzhou, Zhejiang 310003 ; ${ }^{2}$ State Key Laboratory and Collaborative Innovation Center for Diagnosis and Treatment of Infectious Diseases, First Affiliated Hospital, School of Medicine, Zhejiang University, Hangzhou, Zhejiang 310003; ${ }^{3}$ Division of Hepatobiliary and Pancreatic Surgery, First Affiliated Hospital, School of Medicine, Zhejiang University, Hangzhou, Zhejiang 310003 ; ${ }^{4}$ Department of Surgical Intensive Care Unit, First Affiliated Hospital, School of Medicine, Zhejiang University, Hangzhou, Zhejiang 310003, P.R. China
\end{abstract}

Received September 9, 2017; Accepted October 16, 2017

DOI: 10.3892/ijo.2017.4175

\begin{abstract}
Hepatocellular carcinoma (HCC) is the most common type of primary liver cancer, accounting for one-sixth of all malignant tumors, and the mortality rate of HCC ranks second among all cancer-related deaths. Increasing evidence has recently shown that long non-coding RNAs (lncRNAs) play an important role in cancer occurrence and progression, including HCC. Cancer susceptibility candidate 15 (CASC15), a lncRNA, has been reported to be involved in melanoma progression and phenotype switching. However, the function of CASC15 in human HCC is still unknown. In the present study, we evaluated expression of CASC15 and its potential functions in HCC. The expression of CASC15 in HCC tissues was quantitated by the reverse-transcription quantitative polymerase chain reaction, which showed that CASC15 was overexpressed in 59\% (48/82) of HCC tissues compared with corresponding adjacent normal tissues, and the CASC15 expression level was significantly correlated with metastasis $(\mathrm{P}=0.012)$, tumor size $(\mathrm{P}=0.037)$, and TNM stage $(\mathrm{P}=0.013)$. Kaplan-Meier survival curves showed that high CASC15 expression was associated with poor prognosis in HCC patients $(\mathrm{P}<0.05)$. Moreover, a knockdown model of CASC15 was established, which showed that CASC15 significantly impaired HCC cell proliferation, migration, and invasion. CASC15 knockdown also induced
\end{abstract}

Correspondence to: Dr Weilin Wang, Key Laboratory of Precision Diagnosis and Treatment for Hepatobiliary and Pancreatic Tumor of Zhejiang Province, First Affiliated Hospital, School of Medicine, Zhejiang University, 79 Qingchun Road, Hangzhou, Zhejiang 310003, P.R. China

E-mail:wam@zju.edu.cn

${ }^{*}$ Contributed equally

Key words: hepatocellular carcinoma, long non-coding RNA CASC15, proliferation, migration, invasion cell apoptosis in vitro and impaired tumor growth in vivo. In conclusion, CASC15 plays an important role in the progression of HCC, acting as an oncogene. High expression of CASC15 is correlated with a poor prognosis, suggesting that CASC15 may be a predictive biomarker of HCC.

\section{Introduction}

Hepatocellular carcinoma (HCC) is one of the most common malignant tumors in China and worldwide, accounting for onesixth of all malignant tumors, and the mortality rate of HCC ranks second among all cancer-related deaths (1). China has a large population of HCC patients, accounting for nearly half of the world's total, and approximately 422,100 patients were expected to die from this cancer in 2015 (2). Although surgery, together with other comprehensive treatments, has significantly improved the prognosis of patients with unresectable HCC, effective treatments are still lacking (3). Knowledge of the pathogenic mechanisms of HCC is therefore still in its infancy.

Long non-coding RNAs (IncRNAs), with a size $>200$ nucleotides, lack protein-coding ability (4). Increasing evidence has recently suggested that lncRNAs play important roles in cancer occurrence and progression, by regulating gene expression at the epigenetic, transcriptional, posttranscriptional, and translational levels (5-8). Recently, Lessard et al reported that the lncRNA cancer susceptibility candidate 15 (CASC15) is involved in melanoma progression and phenotype switching (9), while it functions as a tumor suppressor in neuroblastoma (10). However, the function of CASC15 in human HCC is still unknown. We therefore investigated the clinical correlations and biological function of CASC15 in human HCC.

\section{Materials and methods}

Patient data and tissue samples. Eighty-two frozen HCC tumor samples and the corresponding adjacent normal tissues were obtained from patients who underwent $\mathrm{HCC}$ resection 
in our department (Hepatobiliary and Pancreatic Surgery, The First Affiliated Hospital, College of Medicine, Zhejiang University, China) from 2013 to 2015 . No patients had other liver diseases or received preoperative therapy. All tissues were preserved at $-80^{\circ} \mathrm{C}$ until analyzed. Clinical data were collected and checked by two independent physicians. Overall survival was defined as the time from the date of the surgery to death or recurrence. The study was approved by the Human Research Ethics Committee of The First Affiliated Hospital, College of Medicine, Zhejiang University. Written informed consent was obtained from all patients.

Total RNA extraction and reverse transcription quantitative polymerase chain reaction $(R T-q P C R)$ analysis. Total RNA was extracted from tissue samples or cell lines using TRIzol ${ }^{\circledR}$ reagent (Invitrogen, Carlsbad, CA, USA) according to the manufacturer's instructions. The RNA concentration and quality were determined by UV spectrophotometry. Reverse transcription of total RNA was performed using the iScript $^{\mathrm{TM}}$ cDNA Synthesis kit (Bio-Rad, Hercules, CA, USA) according to the manufacturer's instructions. The 7500 Fast PCR instrument (Applied Biosystems, Foster City, CA, USA) was used for quantitative PCR amplification. The following primers were used: CASC15 forward, 5'-CACACGCATGGA AAACCCAG-3' and reverse, 5'-GAGGACCTGAGCTGTAA GCC-3'; and glyceraldehyde 3-phosphate dehydrogenase (GAPDH) forward, 5'-AGAAGGCTGGGGCTCATTTG-3' and reverse, 5'-AGGGGCCATCCACAGTCTTC-3' (9).

Cell lines and cell culture. HCC cells (SMMC7721, Hep-G2, Huh-7, HCCLM3, and Hep3B) and human normal hepatocytes (QSG-7701) were maintained at our institute. The cells were maintained in Dulbecco's minimal essential medium or RPMI-1640 containing 10\% fetal calf serum, $100 \mathrm{U} / \mathrm{ml}$ penicillin, and $100 \mu \mathrm{g} / \mathrm{ml}$ streptomycin. All cells were maintained in a humidified incubator at $37^{\circ} \mathrm{C}$ containing $5 \% \mathrm{CO}_{2}$ and were passaged using standard cell culture techniques (11).

The CASC15 knockdown model. SMMC7721 and Huh-7 cells were transfected with siRNA (Smart Silencer; RiboBio, Guangzhou,China) againstCASC15 using Lipofectamine ${ }^{\circledR} 2000$ (Invitrogen) according to the manufacturer's protocols. The siRNA sequences were as follows: locus 1, 5'-CCCTCAGGT GACTACAGAT-3' (sense) and 5'-GCTCAACCACATCTA ATTT-3' (antisense); locus 2, 5'-GCAACATGCTTCACTG TCT-3' (sense) and 5'-GATCGCTGGGAATTCTCCAC-3' (antisense); and locus 3,5'-TCAGAGCTGGCTGCCTGACA-3' (sense) and 5'-GCCAAGAAGAGTATGCAGAG-3' (antisense). CASC15-specific short hairpin (sh-CASC15) RNA lentiviruses were produced using the sequence 5'-GAGCAGATAGCT GAAGAGAGA-3'. After transfection, the cells were treated with $3 \mu \mathrm{g} / \mathrm{ml}$ puromycin (Invitrogen) to establish stable cell lines. Knockdown of CASC15 was confirmed by RT-qPCR.

Cell proliferation assays. Cell proliferation was monitored using the Cell Counting Kit-8 (Dojindo Laboratories, Kumamoto, Japan). Cells $\left(1 \times 10^{4}\right)$ were seeded into 96-well plates after transfection and were counted daily for 3 consecutive days. The absorbance was read at $450 \mathrm{~nm}$. Cell viability was also assessed using the 5-ethynyl-2'-deoxyuridine (EdU) assay (Cell-light EdU Apollo 567 in vitro imaging kit; Ribobio, Guangzhou, China) following the manufacturer's instructions. For the colony formation assay, the cells were inoculated into 6-well plates $(1,000 /$ well $)$ and maintained in a humidified atmosphere at $37^{\circ} \mathrm{C}$ containing $5 \% \mathrm{CO}_{2}$, and the size of the colonies was observed after 2 weeks. Subsequently, the cells were fixed with methanol and stained with $1 \%$ crystal violet.

Cell cycle analysis. The cells were fixed in $70 \%$ ethanol, stored at $4^{\circ} \mathrm{C}$ for $>24 \mathrm{~h}$, stained with DNA Prep (Beckman Coulter, Brea, CA, USA), and analyzed for cell cycle distribution using flow cytometry.

Cell apoptosis analysis. The cells were collected $48 \mathrm{~h}$ after transfection, and then stained with fluorescein isothiocyanate (FITC)-Annexin $\mathrm{V}$ and propidium iodide using the FITC Annexin V Apoptosis Detection kit (BD Biosciences, San Jose, CA, USA) according to the manufacturer's protocol. The stained cells were analyzed using a flow cytometer (Cytomics FC 500; Beckman Coulter, Miami, FL, USA).

Cell migration and invasion assays. To assess cell migration, $4 \times 10^{4}$ transfected cells in $200 \mu \mathrm{l}$ serum-free medium were added to the upper chamber of a Transwell ${ }^{\circledR}$ and incubated for $48 \mathrm{~h}$. The bottom chamber contained $10 \%$ fetal bovine serum. After incubation, the membranes were isolated and stained using DiffQuick (Polyscience, Warrington, PA, USA) according to the manufacturer's protocols. The cells from 10 fields were counted using an inverted microscope (Leica, Malvern, PA, USA). For the cell invasion assay, $40 \mu \mathrm{l}$ diluted Matrigel $^{\mathrm{TM}}$ (1:8) was added to the upper chamber of the Transwell. After incubation at $37^{\circ} \mathrm{C}$ for $30 \mathrm{~min}, 4 \times 10^{4}$ transfected cells in $200 \mu \mathrm{l}$ serum-free medium were added to the upper chamber, and subsequent procedures were performed as described above.

Xenograft model. Five-week-old female BALB/c nude mice were purchased from the Shanghai Sippr/BK Laboratory Animal Co. (Shanghai, China). SMMC7721 cells were collected $48 \mathrm{~h}$ after transfecting with Sh-CASC15 or the control (Shanghai Genechem, Shanghai, China). SMMC7721 cells were suspended in phosphate-buffered saline and injected subcutaneously into the left flank of mice $\left(5 \times 10^{6}\right.$ cells/mouse) . The mice were euthanized after 21 days, and relative data were collected. The tumor volume was calculated using the following formula: volume $=\left(\mathrm{Lx} \mathrm{W}^{2}\right) / 2$, where $\mathrm{L}$ and $\mathrm{W}$ were the longest and shortest diameters of the tumors, respectively. All research involving animals complied with protocols approved by the Zhejiang Medical Experimental Animal Care Commission.

Immunohistochemistry (IHC). Tumors from mice were immunostained with a primary antibody targeting Ki-67 (1:400; Abcam, Cambridge, UK).

Western blot analysis. Western blot analysis was performed as described previously (11). Equal protein loading was monitored using an anti-GAPDH antibody (60004-1-Ig; Proteintech, Rosemount, IL, USA). Primary antibodies against cyclin D1 (ab134175; Abcam), cyclin E1 (ab88259; Abcam), CDK2 
A

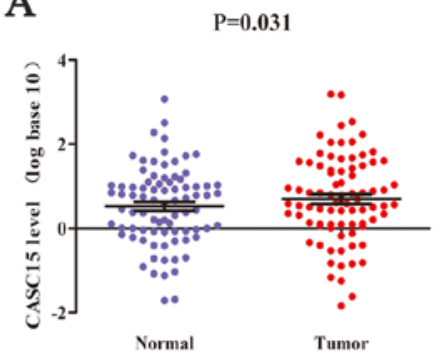

D

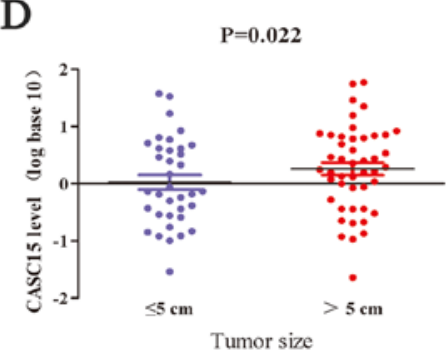

B

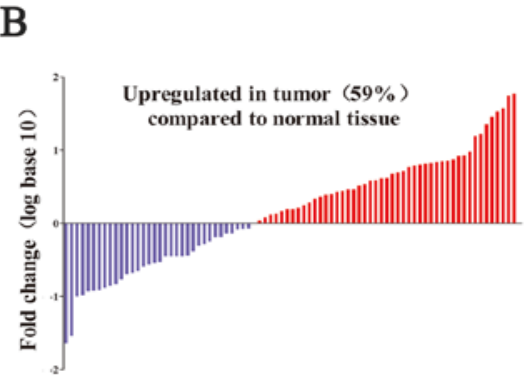

E

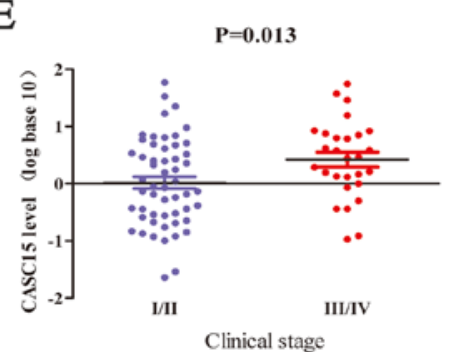

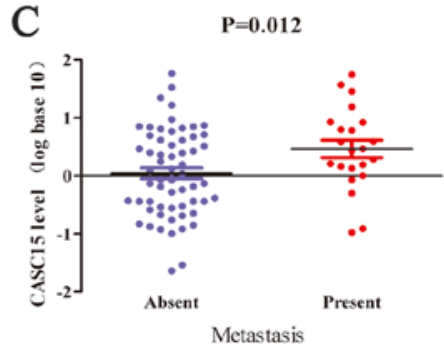

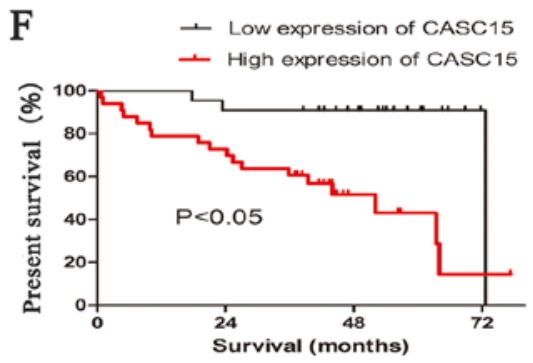

Figure 1. Expression levels and clinical relationships of cancer susceptibility candidate 15 (CASC15) in hepatoma carcinoma (HCC) patients. (A) The relative CASC15 expression level was higher in HCC tissues than in corresponding normal tissues (n=82). (B) CASC15 expression levels were upregulated in most HCC tissues (59\%). (C-E) The expression pattern of CASC15 in HCC according to clinicopathological characteristics. (F) Kaplan-Meier survival curves according to the expression levels of CASC15 in 82 HCC patients.

(ab32146; Abcam), CDK4 (ab137675; Abcam), CDK6 (ab124821; Abcam), caspase 3 (ab17868; Abcam), polyADP-ribose polymerase (PARP) (ab32064; Abcam), Bax (ab32503; Abcam), matrix metalloprotein (MMP)-3, E-cadherin, and N-cadherin (Cell Signaling Technology, Danvers, MA, USA) were used.

Statistical analysis. Data were analyzed using SPSS statistical software for Windows, version 19.0 (IBM, Chicago, IL, USA). The results are presented as means \pm SD. All experiments were performed in triplicate. A value of $\mathrm{P}<0.05$ was considered statistically significant.

\section{Results}

CASC15 is overexpressed in HCC tumor specimens and associated with metastasis, tumor size, TNM stage, and poor prognosis. We determined the expression levels of CASC15 in HCC tissues using RT-qPCR and found that the expression of CASC15 was upregulated in $59 \%$ (48 of 82) of HCC tissues compared with that in paired adjacent normal tissues (Fig. 1A and B). Analyses of potential correlations between CASC15 expression and clinicopathological features in the 82-patient cohort showed that a high expression of CASC15 was significantly correlated with metastasis, large tumor size, advanced and tumor stages (Fig. 1D-F and Table I). To assess an association between CASC15 expression and overall survival rate, the 82 patients with $\mathrm{HCC}$ after hepatectomy were divided into two groups: high CASC15 expression $(n=48)$ and low CASC15 expression $(n=34)$. Kaplan-Meier survival curves showed that high CASC15 expression was associated with poor prognoses in patients with $\mathrm{HCC}(\mathrm{P}<0.05)$.

CASC15 is upregulated in HCC cell lines, promoting tumor cell proliferation and repressing apoptosis in vitro. The expression of CASC15 in human HCC cells (SMMC7721,
Table I. Correlation between CASC15 expression and clinicopathological characteristics with HCC.

\begin{tabular}{|c|c|c|c|}
\hline \multirow[b]{2}{*}{ Clinical parameter } & \multicolumn{2}{|c|}{ CASC-15 } & \multirow[b]{2}{*}{$\mathrm{P}$ value } \\
\hline & $\begin{array}{c}\text { Low no. } \\
\text { cases }\end{array}$ & $\begin{array}{l}\text { High no. } \\
\text { cases }\end{array}$ & \\
\hline Age (years) & & & 0.027 \\
\hline$<50$ & 5 & 18 & \\
\hline$\geq 50$ & 29 & 30 & \\
\hline Sex & & & 0.302 \\
\hline Male & 31 & 47 & \\
\hline Female & 3 & 1 & \\
\hline HBV & & & 0.342 \\
\hline Yes & 31 & 39 & \\
\hline No & 3 & 9 & \\
\hline Cirrhosis & & & 1.000 \\
\hline Yes & 26 & 36 & \\
\hline No & 8 & 12 & \\
\hline AFP & & & 0.122 \\
\hline$\leq 20$ & 20 & 17 & \\
\hline $20-400$ & 3 & 7 & \\
\hline$\geq 400$ & 11 & 24 & \\
\hline Tumor size & & & 0.022 \\
\hline$\leq 5 \mathrm{~cm}$ & 20 & 16 & \\
\hline$>5 \mathrm{~cm}$ & 14 & 32 & \\
\hline TNM stage & & & 0.013 \\
\hline I-II & 28 & 27 & \\
\hline I II-IV & 6 & 21 & \\
\hline Tumor differentiation & & & 0.246 \\
\hline I-II & 14 & 26 & \\
\hline III-IV & 20 & 22 & \\
\hline Metastasis & & & 0.012 \\
\hline Yes & 4 & 18 & \\
\hline No & 30 & 30 & \\
\hline
\end{tabular}


A

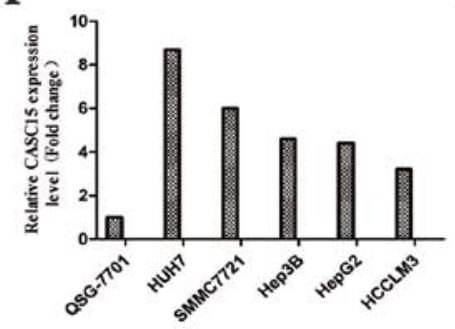

B

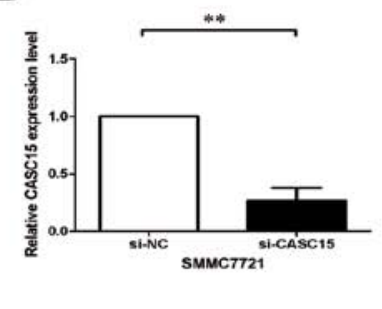

C

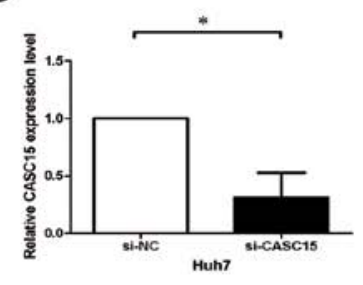

D
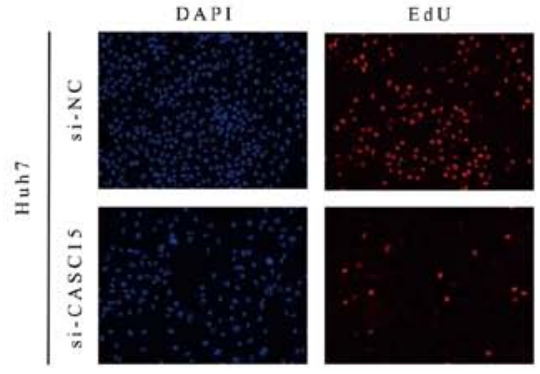

Merge
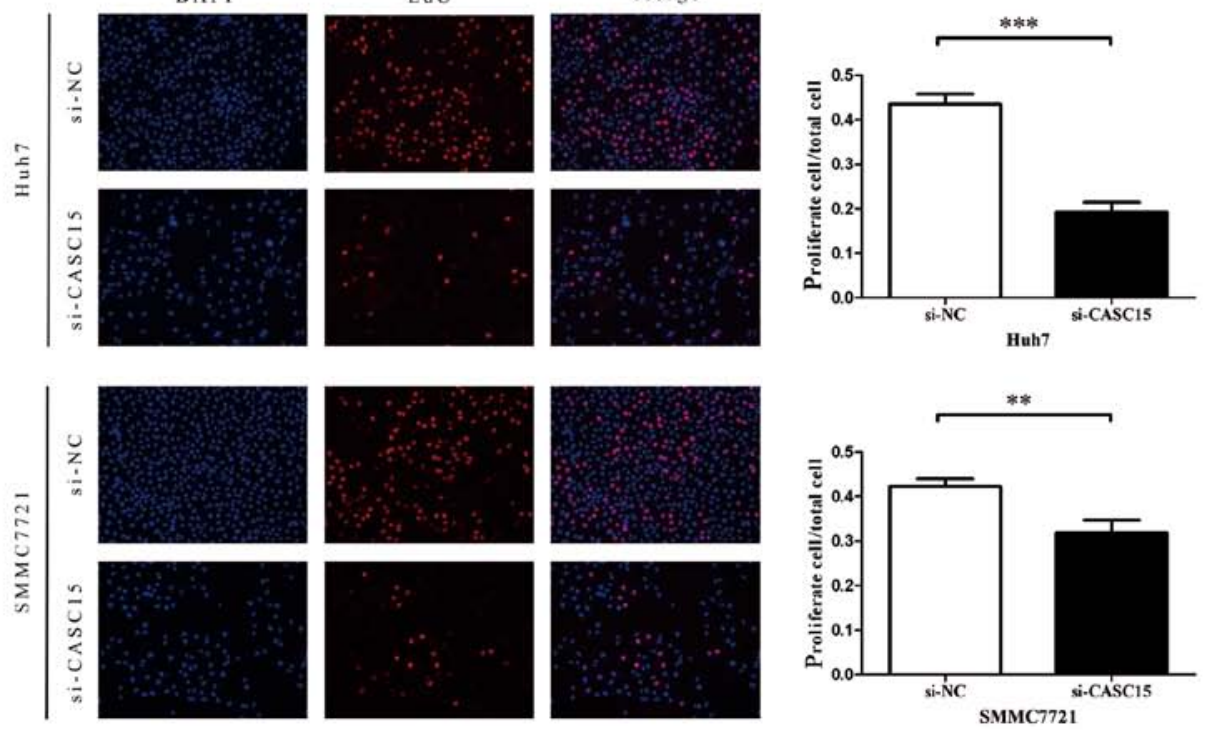

$\mathrm{E}$

Huh-7

SMMC7721
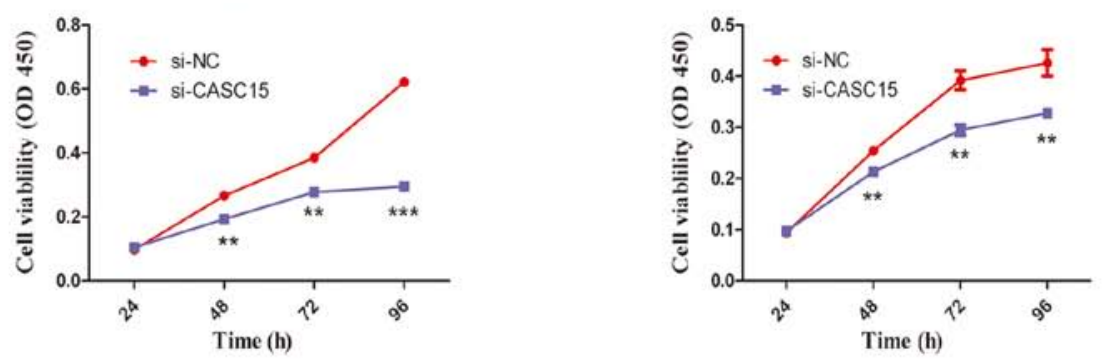

F
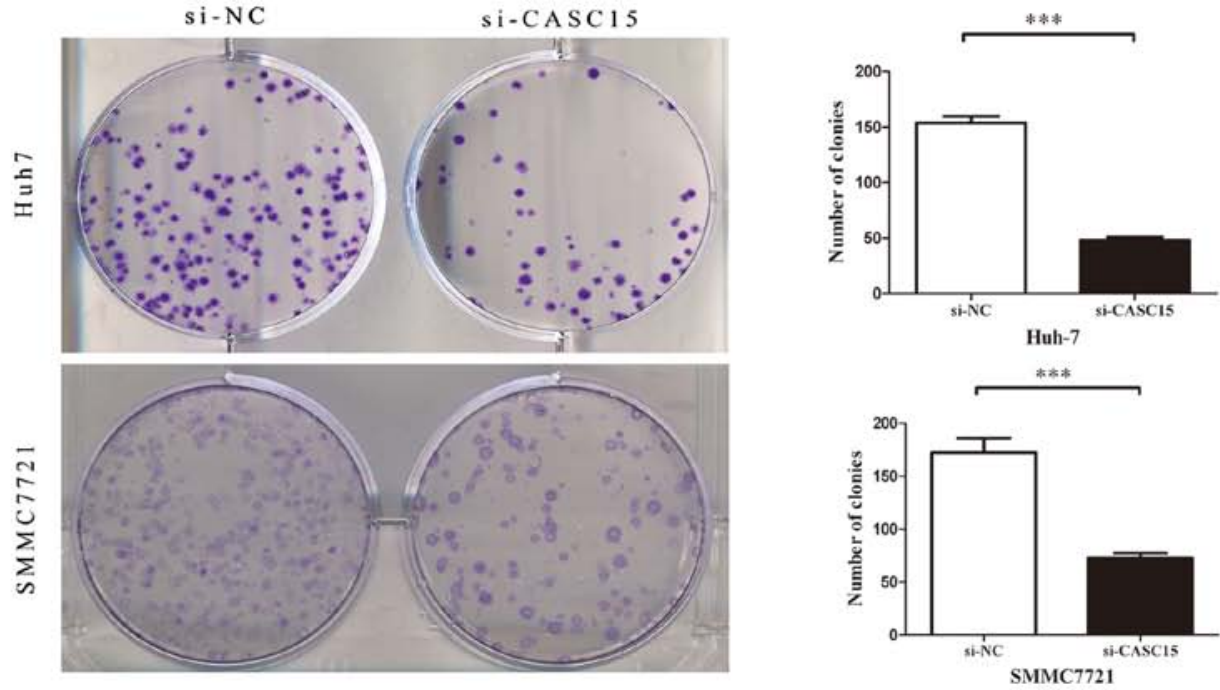

Figure 2. The effects of CASC15 knockdown on HCC cell proliferation. (A) CASC15 was overexpressed in HCC cell lines. (B and C) CASC15 was knocked down by transfection of cells with si-CASC15. (D) The proliferative capacity of HCC cells was assessed by the 5-ethynyl-2'-deoxyuridine assay. (E) Cell Counting Kit-8 assay results in si-CASC15-transfected and control siRNA-transfected Huh7 and SMMC7721 cells. (F) Colony formation assay results in si-CASC15-treated and control Huh7 and SMMC7721 cells. The abbreviations are the same as in Fig. $1 .{ }^{*} \mathrm{P}<0.05,{ }^{* * *} \mathrm{P}<0.01,{ }^{* * * *} \mathrm{P}<0.001$. Magnification, $\mathrm{x} 200$. 

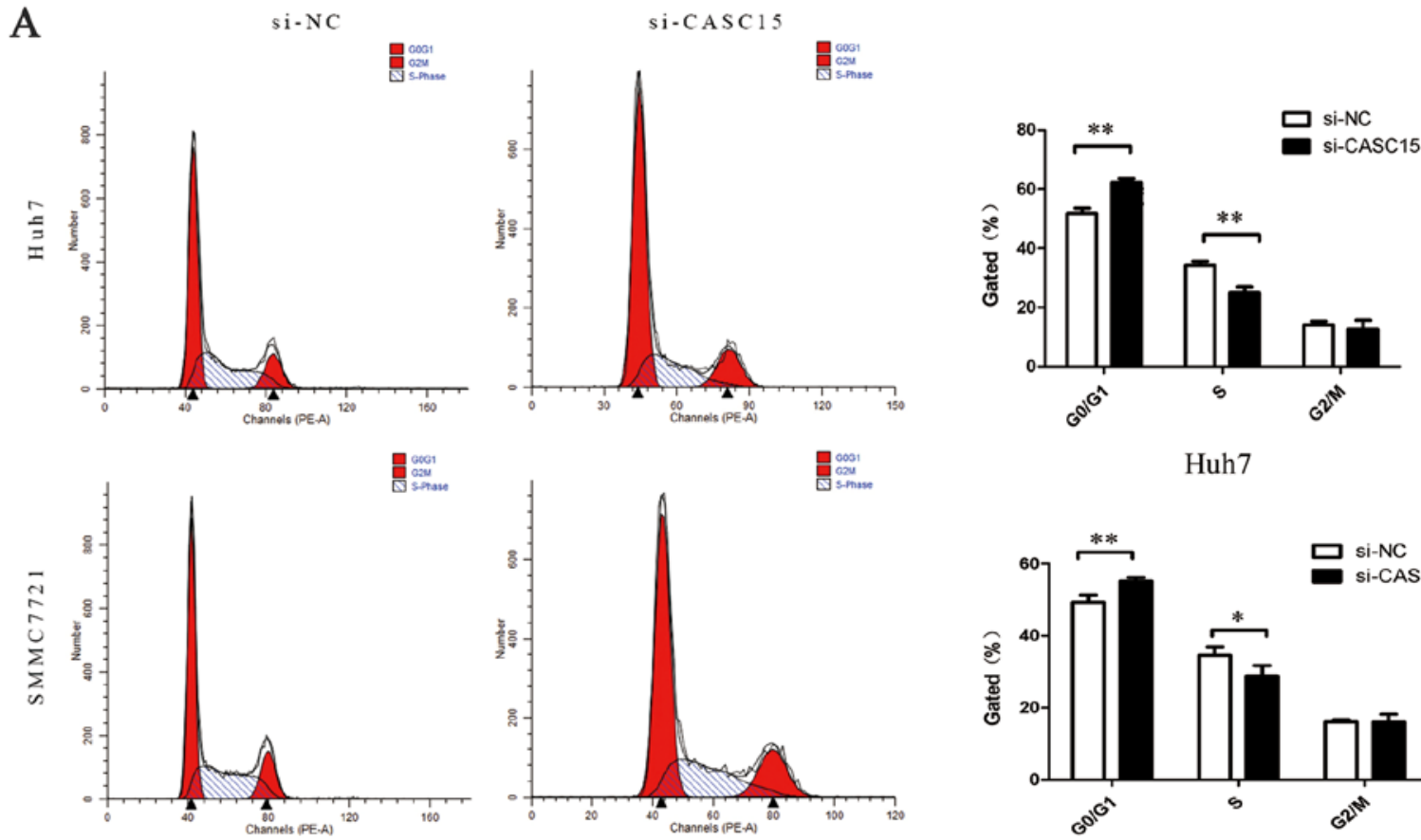

Huh7

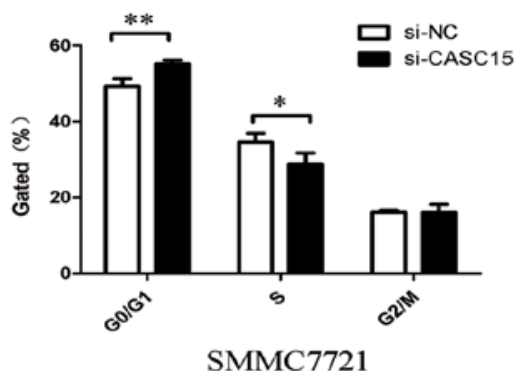

B Huh 7

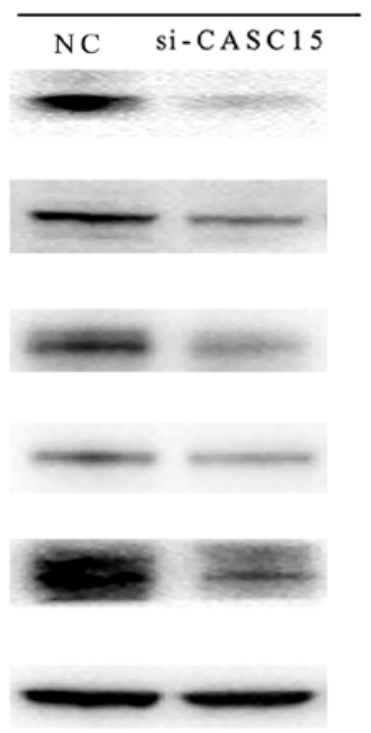

Cyclin D 1
Cyclin E 1
CDK 2
CDK 4
CDK 6
GAPDH

S M MC 7721

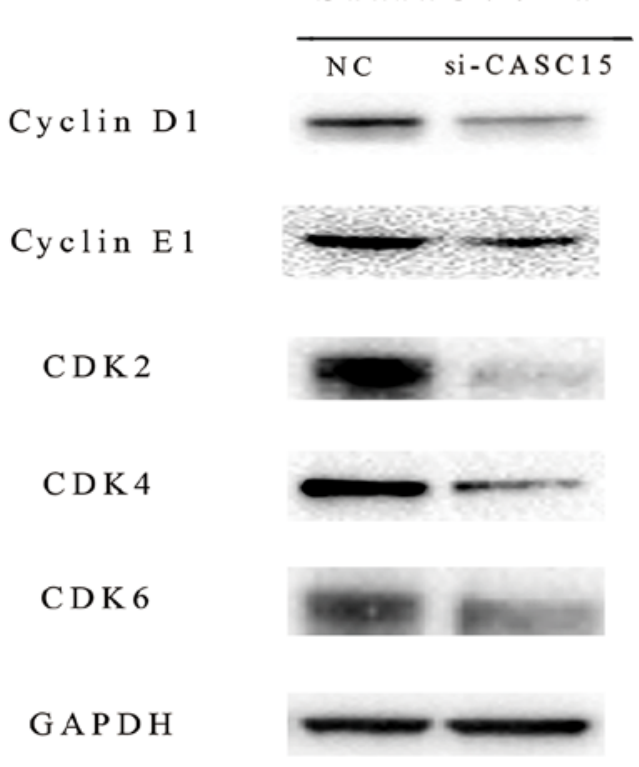

Figure 3. The effects of CASC15 knockdown on the HCC cell cycle. (A) Knockdown of CASC15 increased the number of Huh7 and SMMC7721 cells in $\mathrm{G}_{0} /$ $\mathrm{G}_{1}$ phase. (B) The effect of CASC15 knockdown on the expression of cell cycle-related proteins assessed by western blot analysis. The abbreviations are the same as in Fig. 1. ${ }^{*} \mathrm{P}<0.05,{ }^{* * *} \mathrm{P}<0.01$. FACS, fluorescence-activated cell sorting.

Hep-G2, Huh-7, HCCLM3 and Hep3B) and human normal hepatocytes (QSG-7701) was detected by RT-qPCR. The mRNA level of CASC15 was higher in the HCC cells than in QSG-7701 cells (Fig. 2A). Next, we transfected SMMC-7721 and Huh-7 cells with non-targeting control siRNA or CASC15 siRNA (Fig. 2B and C). An EdU assay performed to quantitate cell proliferation showed that cell proliferation decreased significantly after treatment with the CASC15 siRNA in both Huh7 and SMMC7721 cells (Fig. 2D). This was consistent with the results of the Cell Counting Kit-8 assay (Fig. 2E). In addition, a colony formation assay showed that downregulation of CASC15 significantly inhibited colony formation in
HCC cells (Fig. 2F). To determine if the effect on cell proliferation affected the cell cycle, we assessed cell cycle changes and found that more cells in $\mathrm{G}_{1}$ phase arrest were detected in the CASC15 siRNA-transfected cells compared with the negative control siRNA-transfected cells (Fig. 3A), with decreased expression of cyclin D1, cyclin E1, CDK2, CDK4, and CDK6 compared with the negative control (Fig. 3B). In addition, flow cytometric analyses indicated that knockdown of CASC15 expression increased apoptosis (Fig. 4).

Knockdown of CASC15 inhibits HCC cell migration and invasion in vitro. The role of CASC15 in HCC progression 
A
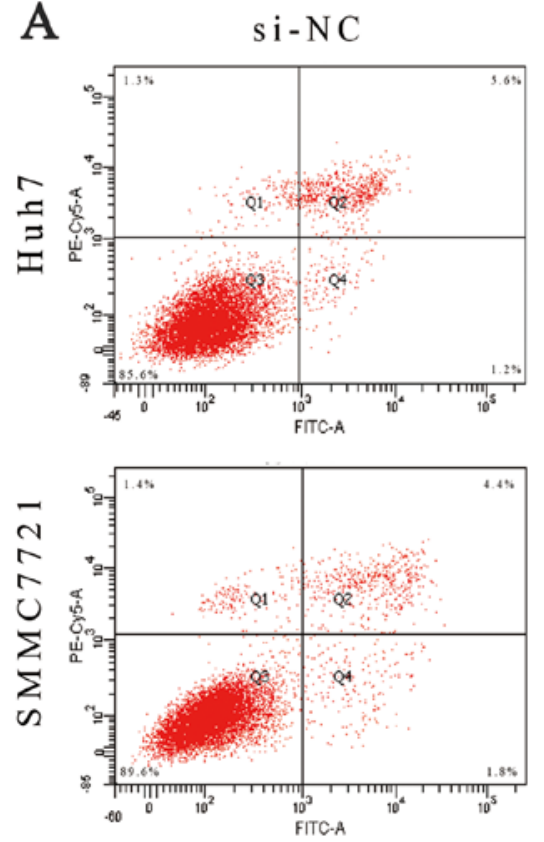

B

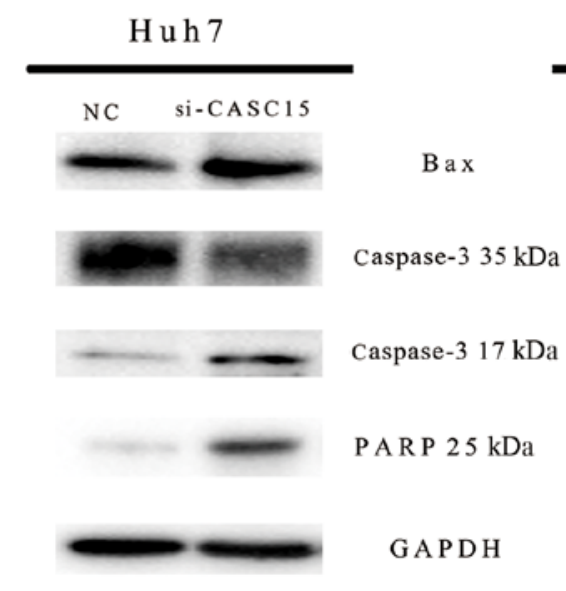

si-C A SC 15
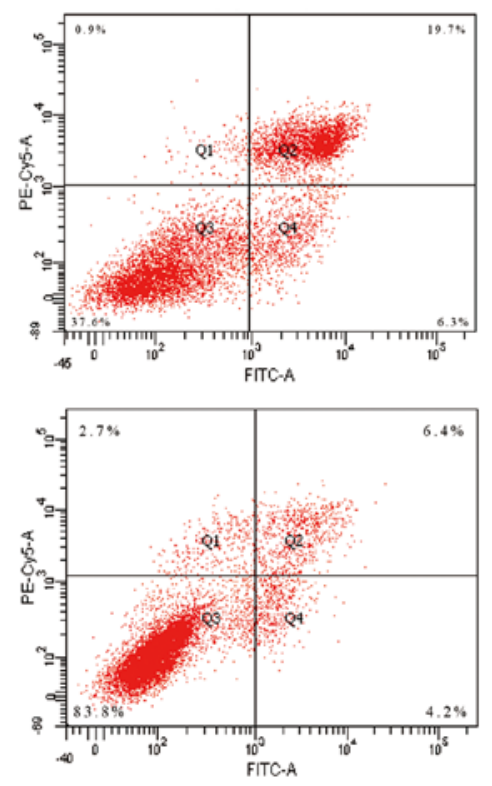

S M M C 7721

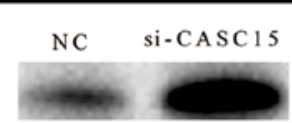

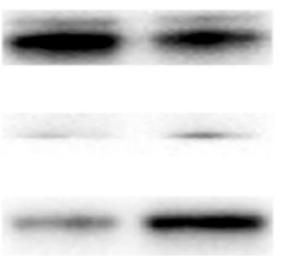

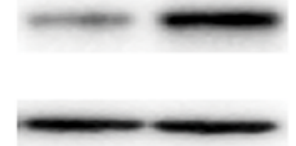

Figure 4. The effects of CASC15 knockdown on HCC cell apoptosis. (A) Knockdown of CASC15 induced apoptosis in Huh7 and SMMC7721 cells as shown by flow cytometry using Annexin V and propidium iodide double staining. (B) The effect of CASC15 knockdown on the expression of apoptosis regulator proteins assessed by western blot analysis. The abbreviations are the same as in Fig. $1 .{ }^{*} \mathrm{P}<0.05,{ }^{* * *} \mathrm{P}<0.01$.

was assessed by Transwell assay to measure cell migration and invasion ability. The results showed that the migration and invasion capabilities of CASC15 siRNA-transfected cells were reduced significantly compared with the negative control siRNA-transfected cells (Fig. 5A and B). Furthermore, western blots showed that E-cadherin expression was increased, while $\mathrm{N}$-cadherin and MMP-3 expression was decreased, in CASC15 siRNA-transfected cells (Fig. 5C).

Knockdown of CASC15 suppressed HCC progression in vivo. To confirm the in vivo oncogenic role of CASC15, we constructed a subcutaneous xenograft model. CASC15 was knocked down in SMMC7721 cells using sh-CASC15 (Fig. 6A). Then, sh-CASC15 and control SMMC7721 cells were injected subcutaneously into nude mice. Consistent with our in vitro results, the sh-CASC15 cells showed significant inhibition of tumor growth compared with the control cells (Fig. 6B and C). The tumor weight of the sh-CASC15-induced tumors was also significantly lower than that of the control tumors (Fig. 6D). Ki-67 staining confirmed
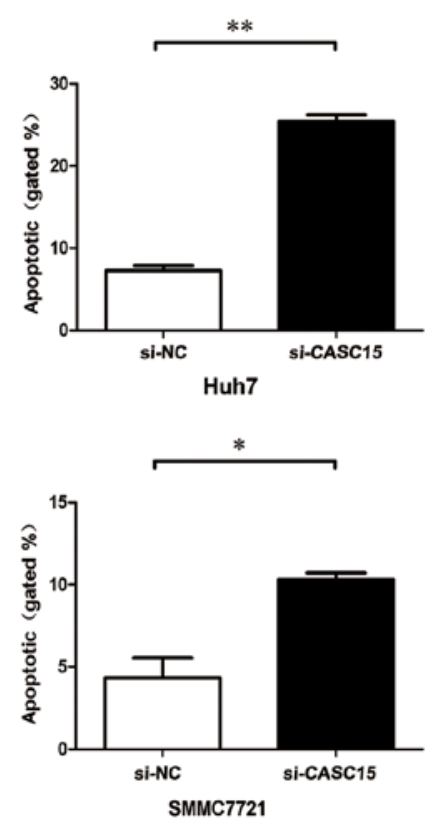

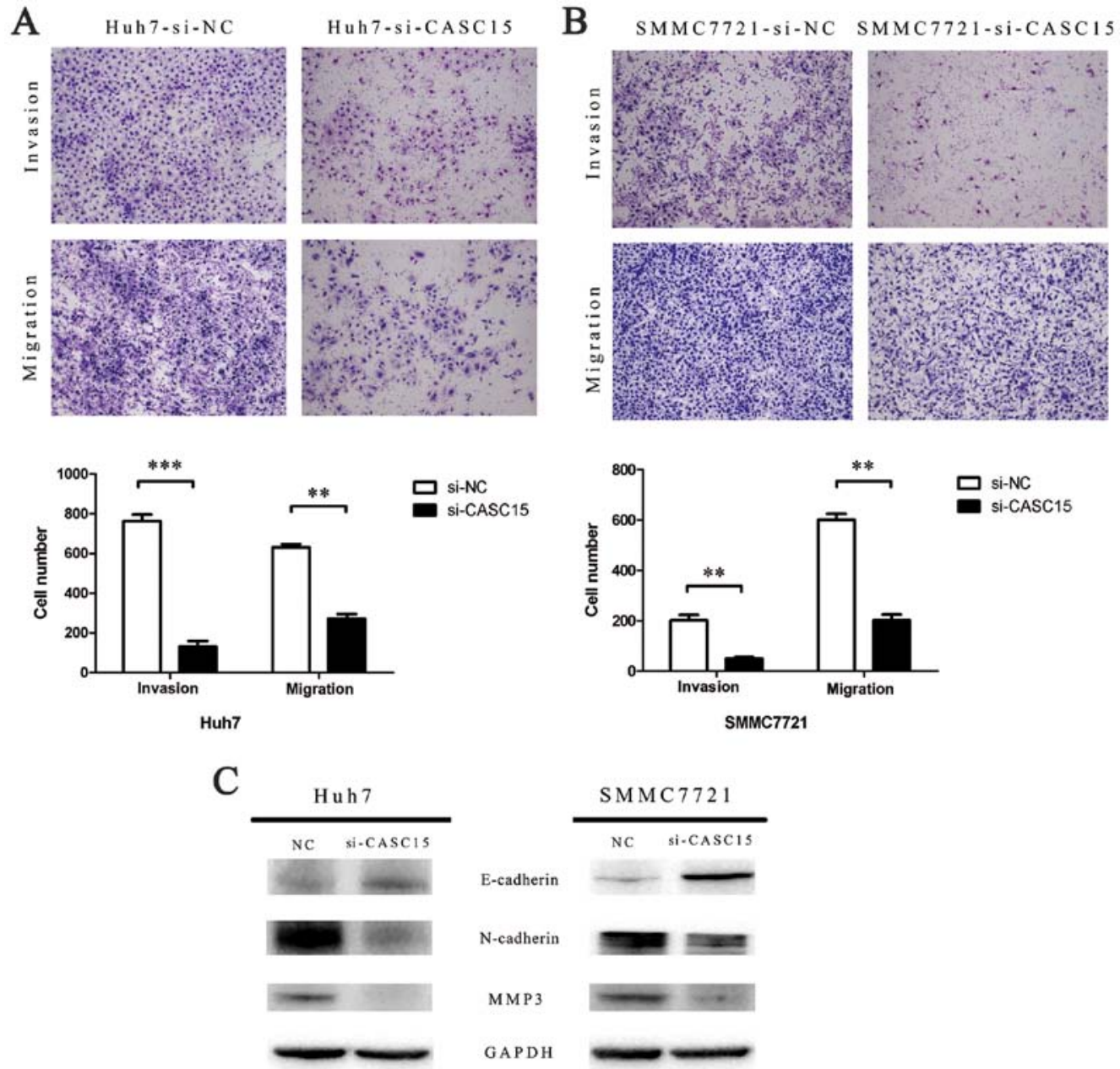

Figure 5. The effects of CASC15 knockdown on HCC cell migration and invasion. (A and B) Migration and invasion assays were performed to detect the effects of CASC15 knockdown on tumor metastasis. (C) The effects of CASC15 knockdown on the expression of epithelial-mesenchymal transition (EMT) proteins assessed by western blot analysis. The abbreviations are the same as in Fig. $1 .{ }^{* *} \mathrm{P}<0.01,{ }^{* * * *} \mathrm{P}<0.001$. Magnification, $\mathrm{x} 40$.

A

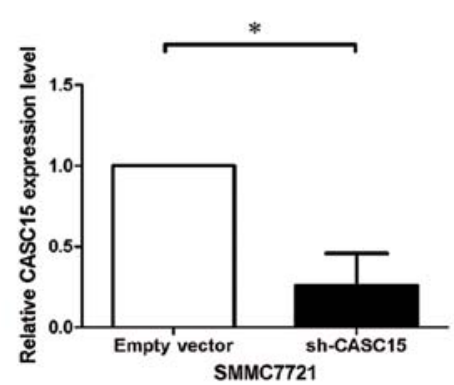

C

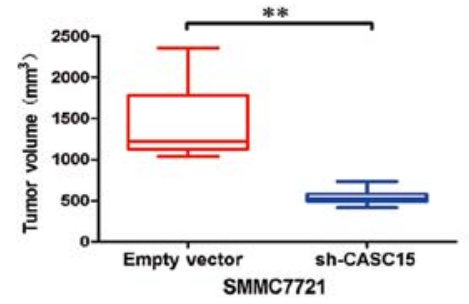

B

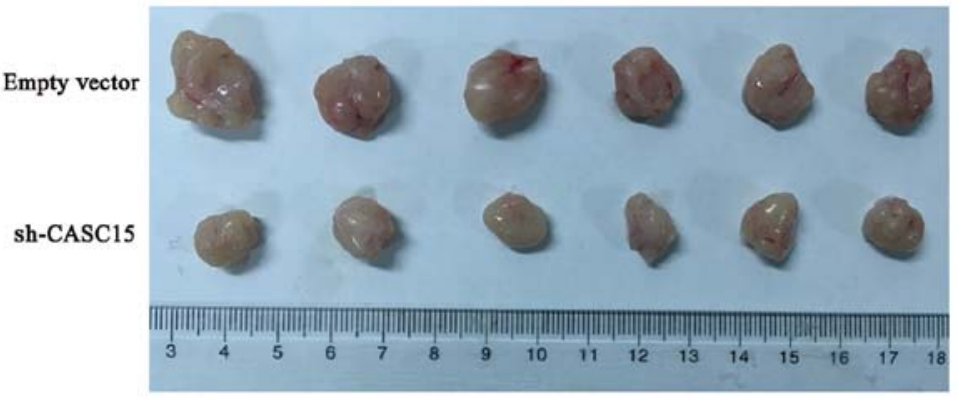

E

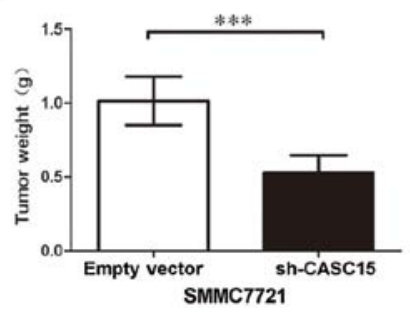

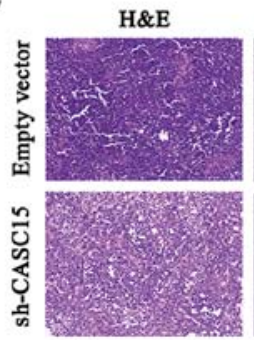

$\mathrm{Ki}-67$

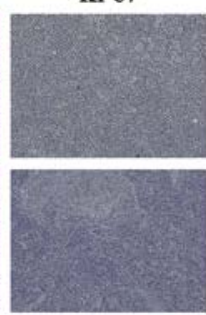

Figure 6. The effects of CASC15 knockdown on tumor growth in vivo. (A) The CASC15 expression level was determined by reverse-transcription quantitative polymerase chain reaction after transfection of SMMC7721 cells with sh-CASC15. (B) Tumors from the nodes of mice 21 days after injection of SMMC7721 cells stably transfected with sh-CASC15 or empty vector. (C) The tumor volumes are expressed as means \pm SD. (D) The tumor weights are expressed as means \pm SD. (E) Representative image of immunohistochemical staining of the tumor. The sh-CASC15 group showed lower Ki-67 protein levels compared with the control. Left, hematoxylin and eosin staining. Right, immunostaining. The abbreviations are the same as in Fig. $1 .{ }^{*} \mathrm{P}<0.05,{ }^{* * *} \mathrm{P}<0.01,{ }^{* * *} \mathrm{P}<0.001$. Magnification, $\mathrm{x} 100$. 
abnormal lipid metabolism to promote HCC progression (19). Many other lncRNAs, such as HOTTIP, HEIH, and DREH, have been reported to play important roles in HCC (20-22).

In the present study, we identified another lncRNA upregulated in HCC, CASC15, which was overexpressed in HCC tissues compared with corresponding adjacent normal tissues. While CASC15 has many isoforms, which may have unique functions (9), our findings showed that their globally upregulated expression was correlated with metastasis, tumor size, TNM stage, and survival outcomes, suggesting that CASC15 may be used as a predictive molecular marker for HCC prognosis. We also found CASC15 consistently upregulated in HCC cell lines, acting as an oncogene in HCC.

Knockdown of CASC15 in SMMC7721 and Huh7 cells resulted in an increase in the number of cells in $\mathrm{G}_{1}$ phase arrest. Cell cycle-related proteins (cyclins E1/D1 and CDK2/4/6) were decreased after CASC15 knockdown. In previous studies, when CDK2 was bound to cyclin E, the complex promoted $\mathrm{G}_{1} / \mathrm{S}$ transition (23), and cyclin D bound to CDK4 and CDK6 led to phosphorylation and inactivation of the retinoblastoma protein, to facilitate E2F-dependent transcription (24). Thus, CASC15 may facilitate proliferation via upregulation of cyclins and CDKs.

Defects in apoptosis are closely related to tumor development (25). Apoptosis includes two main pathways: the extrinsic pathway and intrinsic pathways (26). Bcl-2/Bax activates the intrinsic pathway (27). Caspase-3 is the most important of the executioner caspases, and cleaved caspase- 3 cleaves PARP, leading to apoptosis (28). In our study, cell apoptosis increased with increasing expression of apoptosis-related proteins (Bax cleaved caspase- 3 and PARP) after knockdown of CASC15 expression. Thus, CASC15 may facilitate proliferation by inhibiting the intrinsic apoptosis pathway.

Many studies have reported that the epithelial-mesenchymal transition (EMT) is closely associated with the ability of tumor cells to migrate to distant organs $(29,30)$. Tumor cells modulate their phenotype by activation of various EMT-associated pathways (31). In the present study, cell migration and invasion ability were suppressed by increased expression of an epithelial marker protein (E-cadherin) and decreased expression of a mesenchymal marker protein (N-cadherin), after knockdown of CASC15 expression. Thus, CASC15 facilitates cell migration and invasion by affecting the EMT-associated pathway.

In conclusion, this study is the first to show that CASC15 is overexpressed in HCC tissues compared with corresponding adjacent normal tissues. Furthermore, it showed that high expression of CASC15 is correlated with a poor prognosis. CASC15 promoted HCC proliferation by regulation of apoptosis and the cell cycle, both in vitro and in vivo. Moreover, CASC15 facilitated cell migration and invasion by affecting the EMT-associated pathway. These results indicate that CASC15 acts as an oncogene in HCC and is a potential predictive biomarker for HCC.

\section{Acknowledgements}

This study was supported by National Natural Science Foundation of China [grant no. 81572307(WLW)], Major Project of Medical and Health Technology Development
Program in Zhejiang Province [grant no. 7211902 (WLW)], National Key Basic Research Development Program (973 Program) [grant no. 2013CB531403 (WLW)] and Science and Technology Major Project of Zhejiang Province [grant no. 2014C13G2010059 (WLW)].

\section{References}

1. Stewart BW and Wild CP (eds.): World Cancer Report. Organization World Health, 2014. https://shop.iarc.fr/products/ wcr2014

2. Chen W, Zheng R, Baade PD, Zhang S, Zeng H, Bray F, Jemal A, Yu XQ and He J: Cancer statistics in China, 2015. CA Cancer J Clin 66: 115-132, 2016.

3. Llovet JM and Bruix J: Systematic review of randomized trials for unresectable hepatocellular carcinoma: Chemoembolization improves survival. Hepatology 37: 429-442, 2003.

4. Ponting CP, Oliver PL and Reik W: Evolution and functions of long non-coding RNAs. Cell 136: 629-641, 2009.

5. Zhang ZZ, Shen ZY, Shen YY, Zhao EH, Wang M, Wang CJ, $\mathrm{Cao} \mathrm{H}$ and $\mathrm{Xu} \mathrm{J}$ : HOTAIR long non-coding RNA promotes gastric cancer metastasis through suppression of poly $\mathrm{r}(\mathrm{C})$ binding protein (PCBP) 1. Mol Cancer Ther 14: 1162-1170, 2015.

6. Nie FQ, Sun M, Yang JS, Xie M, Xu TP, Xia R, Liu YW, Liu XH, Zhang EB, Lu KH, et al: Long non-coding RNA ANRIL promotes non-small cell lung cancer cell proliferation and inhibits apoptosis by silencing KLF2 and P21 expression. Mol Cancer Ther 14: 268-277, 2015.

7. Li L, Chen H, Gao Y, Wang YW, Zhang GQ, Pan SH, Ji L, Kong R, Wang G, Jia $\mathrm{YH}$, et al: Long non-coding RNA MALAT1 promotes aggressive pancreatic cancer proliferation and metastasis via the stimulation of autophagy. Mol Cancer Ther 15: 2232-2243, 2016.

8. Wilusz JE, Sunwoo H and Spector DL: Long non-coding RNAs: Functional surprises from the RNA world. Genes Dev 23: 1494-1504, 2009

9. Lessard L, Liu M, Marzese DM, Wang H, Chong K, Kawas N, Donovan NC, Kiyohara E, Hsu S, Nelson N, et al: The CASC15 long Intergenic non-coding RNA locus is involved in melanoma progression and phenotype switching. J Invest Dermatol 135: 2464-2474, 2015.

10. Russell MR, Penikis A, Oldridge DA, Alvarez-Dominguez JR, McDaniel L, Diamond M, Padovan O, Raman P, Li Y, Wei JS, et al: CASC15-S is a tumor suppressor IncRNA at the $6 \mathrm{p} 22$ neuroblastoma susceptibility locus. Cancer Res 75: 3155-3166, 2015.

11. Wang W, Xie Q, Zhou X, Yao J, Zhu X, Huang P, Zhang L, Wei J, Xie H, Zhou L, et al: Mitofusin-2 triggers mitochondria $\mathrm{Ca}^{2+}$ influx from the endoplasmic reticulum to induce apoptosis in hepatocellular carcinoma cells. Cancer Lett 358: 47-58, 2015.

12. Torre LA, Bray F, Siegel RL, Ferlay J, Lortet-Tieulent J and Jemal A: Global cancer statistics, 2012. CA Cancer J Clin 65: 87-108, 2015.

13. Wang KC, Yang YW, Liu B, Sanyal A, Corces-Zimmerman R, Chen Y, Lajoie BR, Protacio A, Flynn RA, Gupta RA, et al: A long non-coding RNA maintains active chromatin to coordinate homeotic gene expression. Nature 472: 120-124, 2011.

14. Tang J, Jiang R, Deng L, Zhang X, Wang K and Sun B: Circulation long non-coding RNAs act as biomarkers for predicting tumorigenesis and metastasis in hepatocellular carcinoma. Oncotarget 6: 4505-4515, 2015.

15. Wang J, Liu X, Wu H, Ni P, Gu Z, Qiao Y, Chen N, Sun F and Fan Q: CREB up-regulates long non-coding RNA, HULC expression through interaction with microRNA-372 in liver cancer. Nucleic Acids Res 38: 5366-5383, 2010.

16. Tu ZQ, Li RJ, Mei JZ and Li XH: Down-regulation of long non-coding RNA GAS5 is associated with the prognosis of hepatocellular carcinoma. Int J Clin Exp Pathol 7: 4303-4309, 2014.

17. Huang MD, Chen WM, Qi FZ, Xia R, Sun M, Xu TP, Yin L, Zhang EB, De W and Shu YQ: Long non-coding RNA ANRIL is upregulated in hepatocellular carcinoma and regulates cell apoptosis by epigenetic silencing of KLF2. J Hematol Oncol 8: 50, 2015.

18. Panzitt K, Tschernatsch MM, Guelly C, Moustafa T, Stradner M, Strohmaier HM, Buck CR, Denk H, Schroeder R, Trauner M, et al: Characterization of HULC, a novel gene with striking up-regulation in hepatocellular carcinoma, as non-coding RNA. Gastroenterology 132: 330-342, 2007. 
19. Cui M, Xiao Z, Wang Y, Zheng M, Song T, Cai X, Sun B, Ye L and Zhang X: Long non-coding RNA HULC modulates abnormal lipid metabolism in hepatoma cells through an miR-9-mediated RXRA signaling pathway. Cancer Res 75: 846-857, 2015.

20. Huang JF, Guo YJ, Zhao CX, Yuan SX, Wang Y, Tang GN Zhou WP and Sun SH: Hepatitis B virus X protein ( $\mathrm{HBx}$ )-related long non-coding RNA (lncRNA) down-regulated expression by $\mathrm{HBx}$ (Dreh) inhibits hepatocellular carcinoma metastasis by targeting the intermediate filament protein vimentin. Hepatology 57: 1882-1892, 2013

21. Yang F, Zhang L, Huo XS, Yuan JH, Xu D, Yuan SX, Zhu N, Zhou WP, Yang GS, Wang YZ, et al: Long non-coding RNA high expression in hepatocellular carcinoma facilitates tumor growth through enhancer of zeste homolog 2 in humans. Hepatology 54: $1679-1689,2011$.

22. Quagliata L, Matter MS, Piscuoglio S, Arabi L, Ruiz C, Procino A, Kovac M, Moretti F, Makowska Z, Boldanova T, et al: Long non-coding RNA HOTTIP/HOXA13 expression is associated with disease progression and predicts outcome in hepatocellular carcinoma patients. Hepatology 59: 911-923, 2014

23. Tsai LH, Harlow E and Meyerson M: Isolation of the human cdk2 gene that encodes the cyclin A- and adenovirus E1A-associated p33 kinase. Nature 353: 174-177, 1991.
24. Massagué J: G1 cell-cycle control and cancer. Nature 432: 298-306, 2004.

25. Kerr JF, Wyllie AH and Currie AR: Apoptosis: A basic biological phenomenon with wide-ranging implications in tissue kinetics. Br J Cancer 26: 239-257, 1972.

26. Elmore S: Apoptosis: A review of programmed cell death. Toxicol Pathol 35: 495-516, 2007.

27. Gu W, Li C, Yin W, Guo Z, Hou X and Zhang D: Shen-fu injection reduces postresuscitation myocardial dysfunction in a porcine model of cardiac arrest by modulating apoptosis. Shock 38: 301-306, 2012.

28. Lazebnik YA, Kaufmann SH, Desnoyers S, Poirier GG and Earnshaw WC: Cleavage of poly(ADP-ribose) polymerase by a proteinase with properties like ICE. Nature 371: 346-347, 1994.

29. Thiery JP, Acloque H, Huang RY and Nieto MA: Epithelialmesenchymal transitions in development and disease. Cell 139: 871-890, 2009.

30. Thiery JP: Epithelial-mesenchymal transitions in tumour progression. Nat Rev Cancer 2: 442-454, 2002.

31. Klymkowsky MW and Savagner P: Epithelial-mesenchyma transition: A cancer researcher's conceptual friend and foe. Am J Pathol 174: 1588-1593, 2009. 